\title{
Error Analysis of Line of Sight Estimation using Purkinje Images for Eye-based Human-Computer Interaction: $\mathrm{HCI}$
}

\author{
Kohei Arai ${ }^{1}$ \\ ${ }^{1}$ Graduate School of Science and Engineering \\ Saga University \\ Saga City, Japan
}

\begin{abstract}
Error analysis of line of sight estimation using Purkinje images for eye-based Human-Computer Interaction: HCI is conducted. Double Purkinje images which are obtained by two points of light sources are used in the proposed method for eye rotation angle estimation. It aimed at the improvement of the eyeball rotation angle accuracy by simply presuming the value of the curvature radius of the cornea. This technique is a cancellation of the time of the calibration that is the problem of past glance presumption. By presuming the size of the radius of curvature of the cornea. As a result, the eyeball rotation angle presumption accuracy of about $0.98 \mathrm{deg}$ was obtained $0.57 \mathrm{deg}$ and horizontally in the vertical direction without doing the calibration.
\end{abstract}

Keywords-Computer input just by sight; Computer input by human eyes only; Purkinje image

\section{INTRODUCTION}

As for the computer input system with human eyes only based on an image-analysis method, many methods have being proposed so far. Matsuda et al. makes the line of sight which connects eyeball rotation center coordinates and a pupil center, and is performing gaze measurement [1]. Eyeball rotation center coordinates are searched for by moving an eyeball in the various directions before gaze measurement. Therefore, since there is no necessity of showing an index, gaze measurement is possible in all places, but preparation takes time and there is a fault of not permitting head movement. Moreover, Ono et al. makes the line of sight which connects cornea center-of-curvature coordinates and a pupil center, and is performing gaze measurement [2].

Cornea center-of-curvature coordinates are the light source of one point, and installing a camera on the optical axis, and are searched for using the general cornea radius-of-curvature value. However, since they assumed models, such as Japanese typical eyeball form, the error remained in the direction estimation of a gaze not a little, and if these methods did not perform a calibration, when there were, they did not become. That is, in order to cancel the gaze estimation error based on the gap of a central fovea to an eyeball center, the refraction in a cornea, and the individual difference concerning the form of a cornea, the calibration which draws a gaze correction coefficient needed to be performed by gazing at two or more indices displayed on the display one by one [3]. Moreover, since it was not what permits a motion of a user, the burden has been forced upon the user. The former presumes the point of regard on the display at the point of the look obtained from gaze estimation (three dimension measurement) of not only the direction of a gaze but both eyes, and uses the pupil center and the corneal reflex center for gaze estimation. The latter presumes a cornea center of curvature using two Purkinje images, makes a user gaze at the index of three points, presumes an eyeball rotation center, is the method of making a gaze the straight line which connects these, and is verifying accuracy by the experiment using a model eye [4].

There are some methods which allow gaze estimations and its applications for HCI [5]-[35]. In particular, paper 13 describes the method for gaze detection and line of sight estimation. In this paper, an error analysis is made for the previously proposed method. For the method, an expensive stereo camera is not needed, but only a cheap simple eye camera permits a motion of a user, and the method of determining the direction of a look from a pupil center and a cornea center of curvature is proposed without the calibration which forces a user a gaze of three points. By specifically measuring an eyeball cornea curvature radius simply, the degree estimation of eyeball rotation angle which does not need a calibration is performed, details are extracted from a face picture, the posture of a head is detected from those relative spatial relationships, and a motion of a head is permitted. The light source of two points was used for measurement of the cornea curvature radius of an eyeball, and two Purkinje images obtained from the cornea surface were used for it. At this time, it decided to also use together the near infrared light source which a camera has using the nearinfrared camera which became budget prices, and to acquire the clear Purkinje image in recent years. When five subjects estimate the direction estimation accuracy of a look for this, a motion of the head of the 30 roll directions and the 15 directions of a pitch is permitted, and since it checked that the direction of a gaze could be presumed without a calibration with the error of 0.57 to 0.98 degrees, it reports here.

The angle estimation method of eyeball rotation angle of using two light sources and one near infrared camera is proposed first, the head angle detection method using the details in a face picture is described, and this paper estimates the validity of the proposed method by the direction estimation experiment of a look by five subjective examiners. 
The following section describes the proposed line of sight vector estimation with Purkinje images briefly followed by the proposed error analysis method. Then experimental results are described together with some remarks. Finally, conclusion is described with some discussions and future investigations.

\section{PROPOSED METHOD FOR LINE OF Sight ESTIMATION}

\section{A. Eye model}

Fig.1 (a) shows eye shape model while Fig.1 (b) shows the definitions of Purkinje images of the first to the fourth Purkinje images. The size and the curvature of cornea, sclera, retina, and eyeball are different for everybody. Therefore, calibration is required before using computer input just by sight. It is possible to estimate the size and the curvature by using the locations of the first to the fourth Purkinje images. The line of sight is defined as the line starting from the cornea curvature center which is estimated with Purkinje images to pupil center.

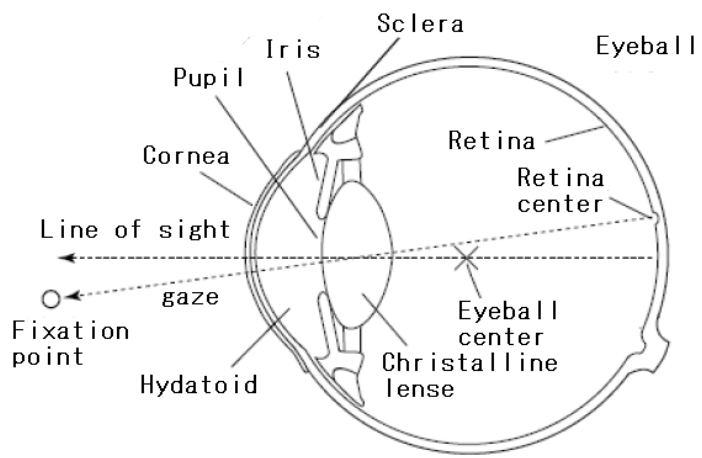

(a)Eye shape model

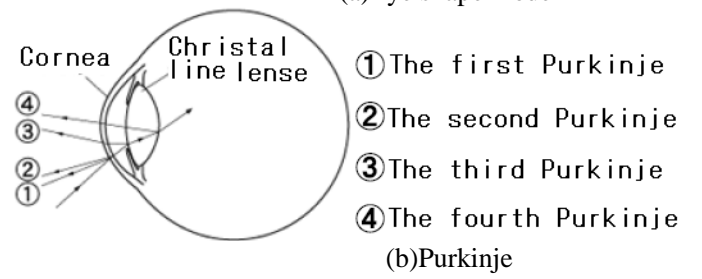

Fig. 1. Eye model and Purkinje images

\section{B. Procedure for estimation of fixation point on display at which user is looking}

The procedure for estimation of fixation point on display at which user is looking is as follows,

1) Cornea curvature radius is estimated with double Purkinje images

2) Pupil center is determined with ellipsoidal approximation of pupil shape

3) Cornea curvature center is determined with geometric relations among eyeball, camera, display and light sources

4) Line of sight is determined with the cornea curvature center and pupil center

5) Fixation point on the display is determined with the line of sight vector

Fig. 2 shows the method for estimation of cornea curvature center and radius.

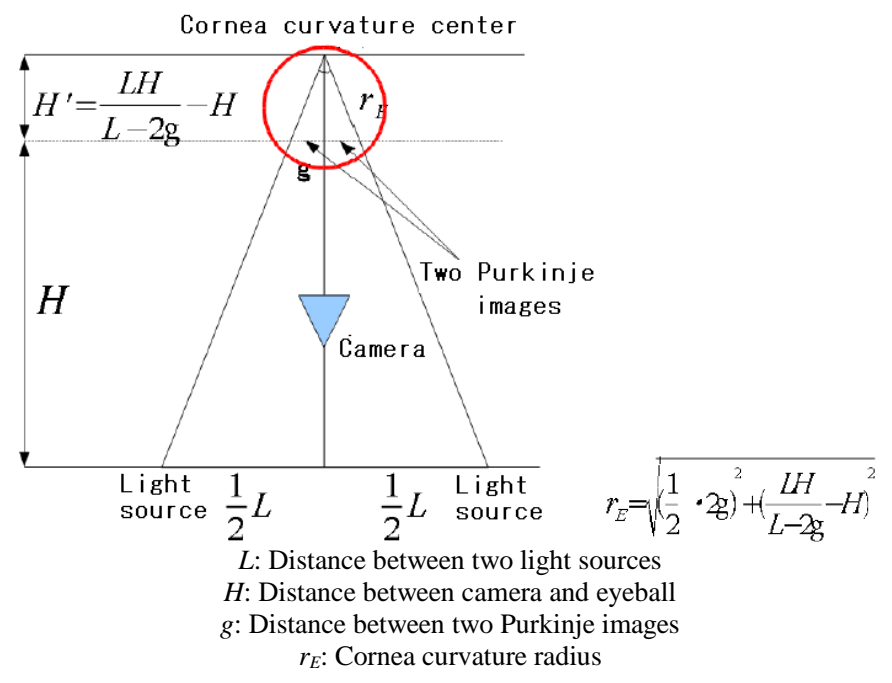

Fig. 2. Method for estimation of cornea curvature center and radius

$\mathrm{L}$ and $\mathrm{H}$ are given. The distance between two Purkinje images can be measured as follows,

1) binarize the acquired NIR image of the eye and its surroundings,

2) isolated noise pixels are removed by using morphological filter,

3) the distance between the locations of two Purkinje images is measured

This procedure is illustrated in the Fig.3. Thus the cornea curvature radius can be estimated.

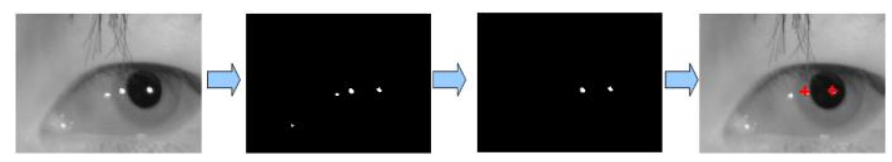

Fig. 3. Procedure of the cornea curvature radius measurement

Through this procedure, the relation between the distance between the eye and the camera and the number of pixels within $10 \mathrm{~cm}$ of distance interval is shown in Fig.4. Meanwhile, the estimated cornea curvature radius is shown in Fig.5 as a function of the distance from the eye. These can be derived from the following equation representing the cornea curvature radius.

$$
r_{E}=\sqrt{\left(\frac{1}{2} \cdot 2 g\right)^{2}+\left(\frac{L H}{L-\partial g}-H\right)^{2}}
$$

Next, a pupillary zone is extracted using a dark pupil method, ellipse approximation of the pupil form is carried out, and a pupil center is searched for. With a dark pupil method, if an infrared floodlight is installed in the position distant from the lens optic axis of a camera and an eyeball is illuminated, the reflection of light of the pupil portion of the illuminated eyeball will be lost, and the pupil portion of the eyeball picture caught with the camera will use the character to become dark here. Image processing extracts this dark portion. 


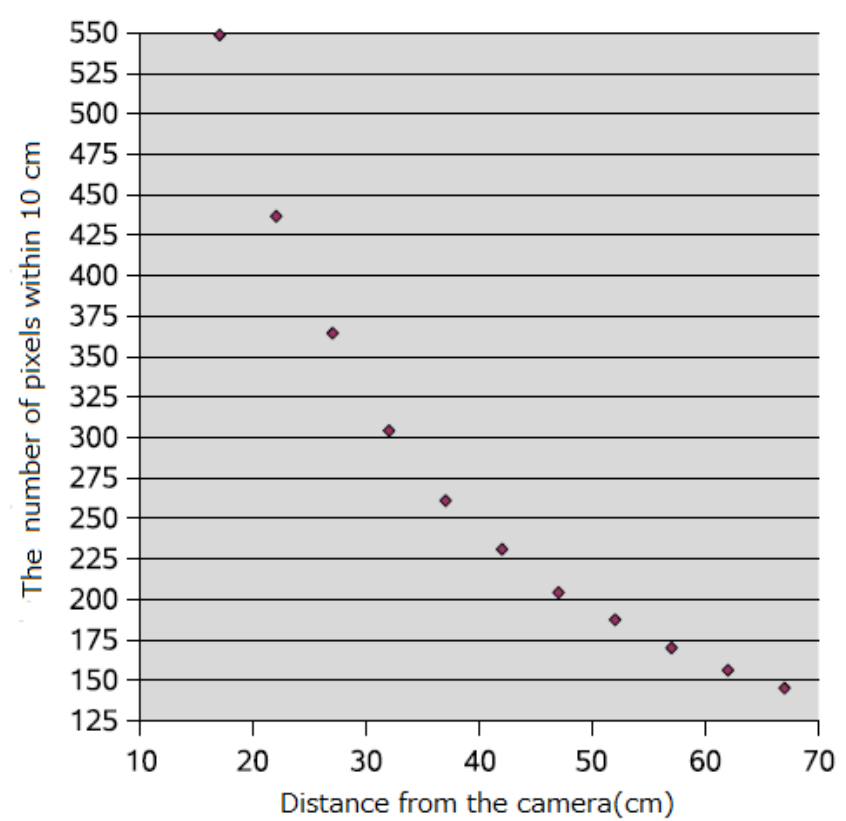

Fig. 4. Relation between the distance between the eye and the camera and the number of pixels within $10 \mathrm{~cm}$ of distance interval

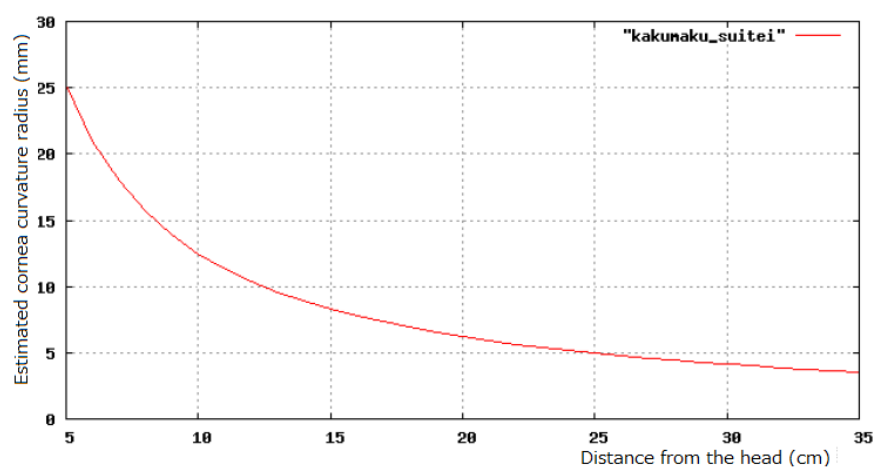

Fig. 5. Relation between the estimated cornea curvature radius and the distance from the eye

Moreover, in the extracted pupillary zone, since reflected lighting and the up-and-down portion of a pupil hide and are missing by the eyelid, the pupil may not be able to be extracted correctly. Therefore, edge is detected from the obtained temporary pupillary zone, and the edge concerned is approximated to an ellipse by a least squares method. However, when it calculates using except a pupil outline, there is a possibility of resembling the mistaken ellipse. Therefore, pupil ellipse approximation using the character of an ellipse was performed.

That is, in Fig.6, straight lines 1, m, and $\mathrm{n}$ shall be parallel, and 1 and $n$ shall exist in the equal distance from $m$. The intersection of an ellipse and a straight line 1 is set to $a$ and $b$, the intersection of an ellipse and a straight line $\mathrm{n}$ is set to $\mathrm{c}$ and $\mathrm{d}$, and the middle point of the intersection of an ellipse and a straight line $\mathrm{m}$ is set to $\mathrm{o}$. When the middle point of a line which connects the middle point of $\mathrm{a}$ and $\mathrm{b}$ and the middle point of $\mathrm{c}$ and $\mathrm{d}$ is set to $\mathrm{o}_{0}$, there is character in which $\mathrm{o}_{0}$ overlaps with 0 .

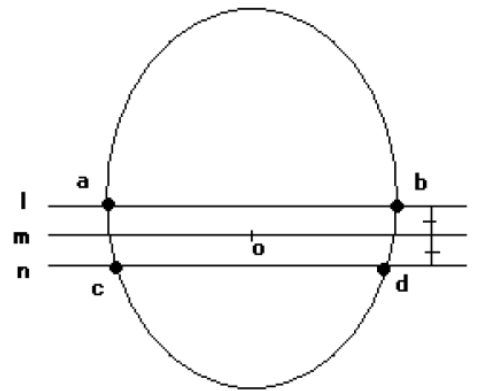

Fig. 6. Elliptic approximation

Least square method for elliptic approximation is illustrated in Fig.7.

$$
\left(\frac{\left(X_{i}-X_{0}\right) \cos \theta+\left(Y_{i}-Y_{0}\right) \sin \theta}{a}\right)^{2}+\left(\frac{-\left(X_{i}-X_{0}\right) \sin \theta+\left(Y_{i}-Y_{0}\right) \cos \theta}{b}\right)^{2}=1
$$
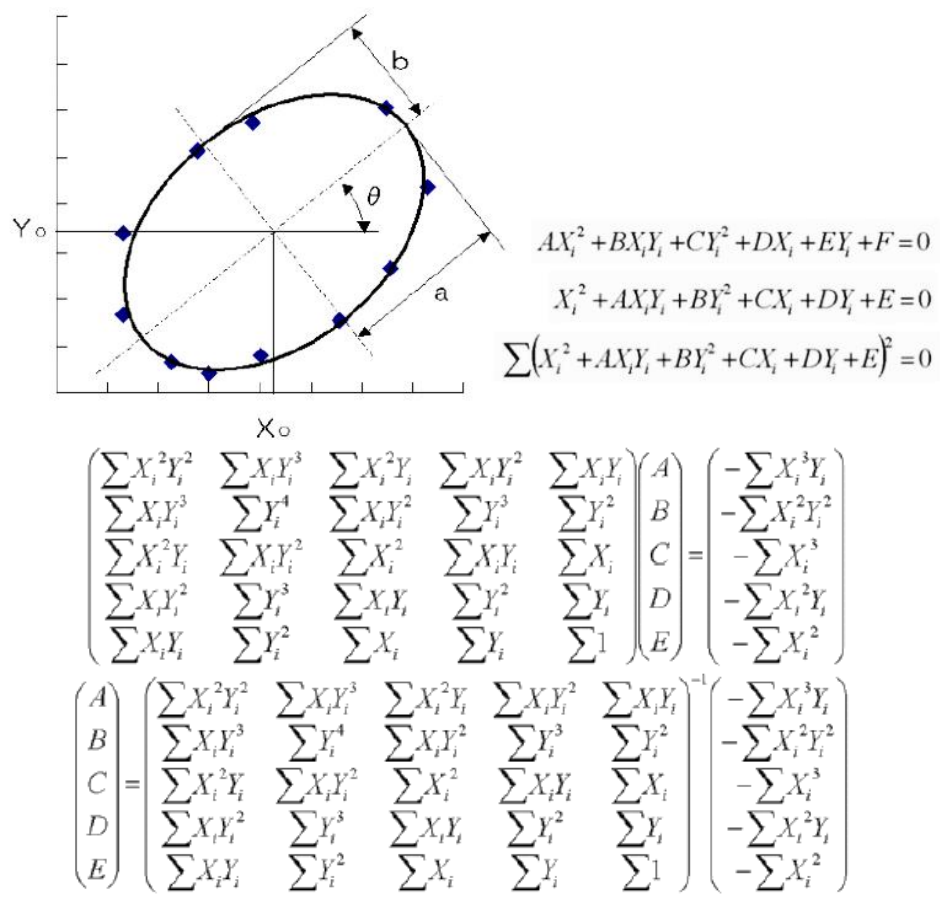

$$
X_{0}=\frac{A D-2 B C}{4 B-A^{2}} \quad Y_{0}=\frac{A C-2 D}{4 B-A^{2}} \quad \theta=\frac{\tan ^{-1}\left(\frac{A}{1-B}\right)}{2}
$$

$a=\sqrt{\left(X_{0} \cos \theta+Y_{0} \sin \theta\right)^{2}-E \cos ^{2} \theta-\left\{\left(X_{0} \sin \theta-Y_{0} \cos \theta\right)^{2}-E \sin ^{2} \theta\right\} \frac{\sin ^{2} \theta-B \cos ^{2} \theta}{\cos ^{2} \theta-B \sin ^{2} \theta}}$

$b=\sqrt{\left.\left(X_{0} \sin \theta-Y_{0} \cos \theta\right)^{2}-E \sin ^{2} \theta-\left(X_{0} \cos \theta+Y_{0} \sin \theta\right)^{2}-E \cos ^{2} \theta\right\} \frac{\cos ^{2} \theta-B \sin ^{2} \theta}{\sin ^{2} \theta-B \cos ^{2} \theta}}$

Fig. 7. Least square method for elliptic approximation

$\mathrm{N}$ which is in the equal distance about a temporary pupillary zone from the straight line $\mathrm{m}$ drawn in the center. The point of hitting $o_{i 0}(i=1-N)$ of the group of the parallel lines is searched for. The point searched for is distributed on a straight line $\mathrm{m}$. When there are few noises enough, the position in which most many points $\mathrm{o}_{0}$ gathered is equivalent to the position of $\mathrm{o}$. Since the point which is distant from the 
position will include the point which is not on the locus of an ellipse, it accepts them. o point $a_{i}$ which is alike and has sufficiently near $o_{i 0} ; b_{i} ; c_{i}$; Since $d_{i}$ is a point on an ellipse, ellipse approximation of an exact pupil outline can be performed by using them.

Next, a cornea center of curvature is searched for from the spatial relationship of the acquired cornea curvature radius and the light source of Fig.8, an eyeball, and a camera, and the geometric expression of relations in a Figure. A cornea center of curvature exists here on the bisector which ties a camera, the Purkinje image, and lighting. It asks for a look vector from a pupil center and a cornea center of curvature.

Finally, the point of regard on a display is computed from a look vector. The vector which passes along two points, a cornea center of curvature and a pupil center, is made into a look vector, and the point of regard on a display is computed.

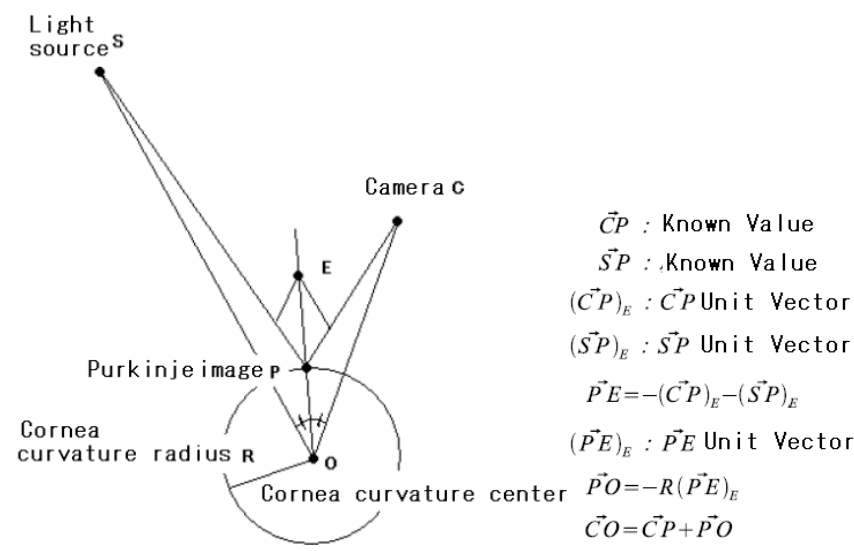

Fig. 8. Geometric relation among light source, camera and eyeball

It is considered as a camera position $(0,0,0)$, and is considered as the main coordinates $(0,0 \mathrm{z})$ of the picture picturized with the camera. If a line of sight vector is made into $\mathrm{v}=\left(\mathrm{x}_{\mathrm{v}} ; \mathrm{y}_{\mathrm{v}} ; \mathrm{z}_{\mathrm{v}}\right)$, a camera, lighting, and a display assume that it is being fixed and the head is also being fixed, and are $\mathrm{z}$. The distance of the direction of an axis is known. When distance of an eyeball and a display is set to $\mathrm{z}_{\mathrm{h}}$, point-of-regard coordinates $\mathrm{t}=$ on a display $\left(\mathrm{x}_{\mathrm{t}} ; \mathrm{y}_{\mathrm{t}}\right)$ is,

$$
\left(\begin{array}{c}
x_{t} \\
y_{t}
\end{array}\right)=\left(\begin{array}{c}
x_{v} \times \frac{z_{h}}{z_{v}} \\
y_{v} \times \frac{z_{h}}{z_{v}}
\end{array}\right)
$$

so that the gaze fixed point can be calculated as is shown in Fig.9.

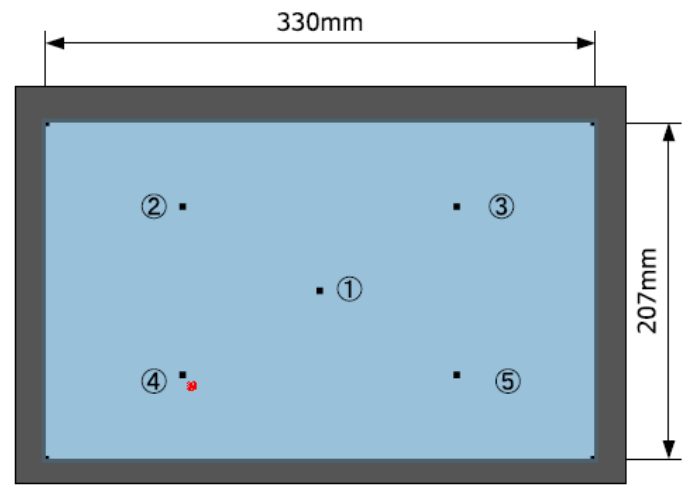

(a) Calibration point locations on the computer screen for evaluation of gaze position estimation accuracy (red point shows the estimated gaze fixed point)

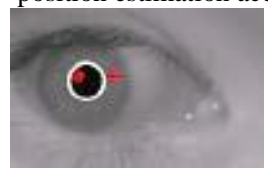

(b) The estimated cornea center and pupil center

Fig. 9. Method for gaze fixed position

During the process, ellipsoidal contour and feature points extraction can be done with the procedure as shown in Fig.10.
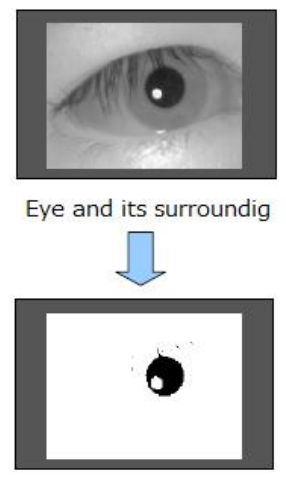

Binarized image
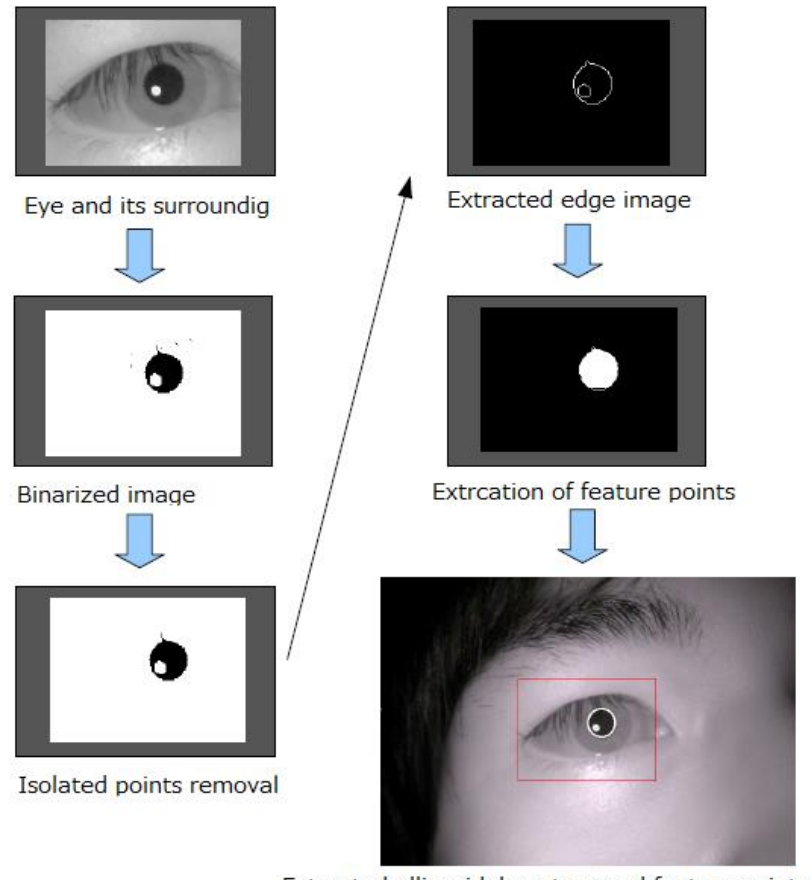

Extracted ellipsoidal contour and feature points

Fig. 10. Procedure for ellipsoidal contour and feature points extraction 


\section{EXPERIMENT FOR ERROR ANALYSIS}

\section{A. Configuration}

Experimental configuration is setup as shown in Fig.11 (a). Meanwhile, the top view of configuration is shown in Fig.11

(b). The measurement equipment used is as follows,

PC: Dell Computer Optiplex 755 Core 2 Quad 2.66 GHz CPU with 2 MB RAM of main memory

\section{OS: WindowsXP home Service Pack2IR}

Camera: (640 by 480 pixels) of DC-NCR 131 type manufactured by NetCowBoy and frame rate: 10 frames-persecond

Infrared floodlight: KMT-7787.

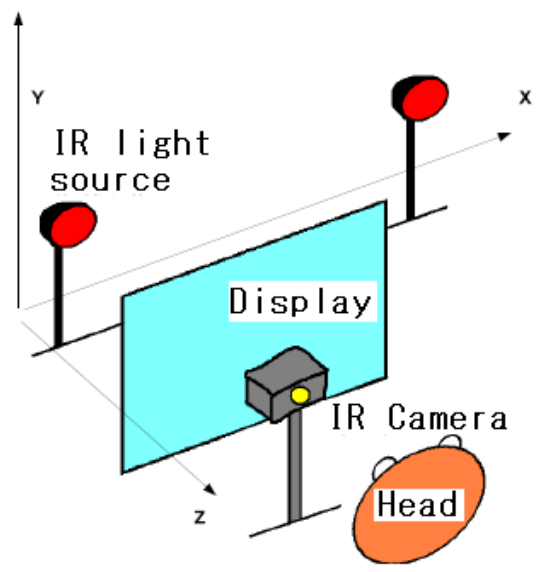

(a)Experimental configuration

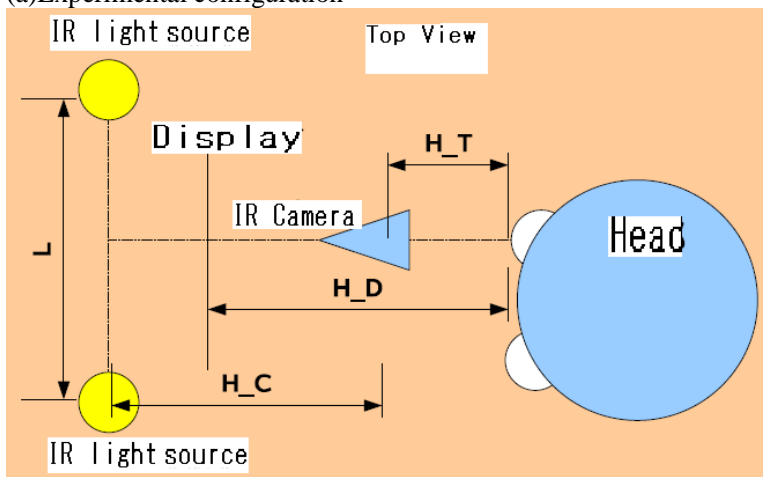

(b)Top view of the configuration

Fig. 11. Experimental configuration for error analysis

The parameters of the experimental configuration are as follows,

$\mathrm{L}=1000 \mathrm{~mm} ; \mathrm{HC}=670 \mathrm{~mm} ; \mathrm{HT}=150 \mathrm{~mm} ; \mathrm{HD}=$ $380 \mathrm{~mm}$

Software development environment is as follows,

Microsoft Visual C++

Microsoft Visual studio.NET2

OpenCV 1.0

Moreover, the picture acquired from the camera on the occasion of look measurement was processed in real time, and performed accuracy verification.

\section{B. Preliminary Experimental Results}

The measurement result of a cornea curvature radius is shown in Fig.12. The spike in a Figure is based on incorrect detection of the Purkinje image, and these can be accepted from measurement of a cornea radius. Moreover, two lines in a Figure are the maximum errors of the cornea curvatureradius point estimate considered when movement of $\pm 1.0 \mathrm{~cm}$ has a head in the display direction. The average of the cornea curvature-radius point estimate except an edge portion is used for the point estimate of the cornea curvature radius used for the actual degree estimation of eyeball rotation angle. The value of a cornea curvature radius was set to $R=7.92 \mathrm{~mm}$ from this result.

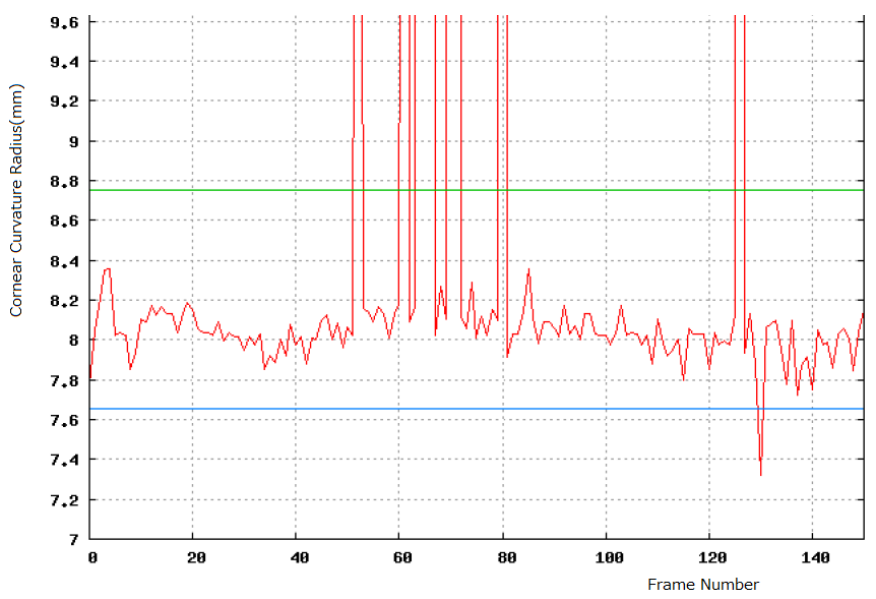

Fig. 12. Result from the preliminary experiment

\section{Sensitivity and Stability}

How to permit a motion of a head finally is shown. The both ends of eye and a mouth, the middle point between two ends of eye, and the middle point of the both ends of a mouth are detected from a face picture. Template matching is used for pursuit of details at these details extraction using OpenCV. By the middle point of both eyes, and the middle point of a mouth, three square shapes can be constituted and a plane can be defined. The normal direction of this plane is judged to be the posture of a head. Once extracting these details, by using template matching which can pursue most in a short time, it is devising so that the pursuit in real time may be possible. An example of details extraction and pursuit is shown in Fig.13. The distance between the details in a Figure is found and the degree of rotation angle of a head can be presumed in comparison with the position of the details extracted in early stages by OpenCV. The blue line segment of Fig.13 is a head posture (the plane normal direction is shown.). Moreover, a green line segment connects the middle point between the middle points of both eyes, and the middle point of the both ends of a mouth.

The geometric relation between the rotation center of a head and an eyeball rotation center is shown in Fig.14, and can presume an eyeball rotation center position after this. If the method of presuming a look from an eyeball rotation center position is considered to be the same thing as the above-mentioned method, a motion of a head will be permitted and the direction estimation of a look will be 
attained. At this time, that the both ends of both eyes and the both ends of a mouth are not occluded are the conditions which presume a head rotation center and an eyeball rotation center, and 30 degrees and about 15 degrees are rotation allowable limits in the roll direction and the direction of a pitch.

As described before, movement in the direction of $\mathrm{z}$ is compared with movement to $\mathrm{x}$ and a $\mathrm{y}$ direction, and has serious influence on eyeball angle detection.
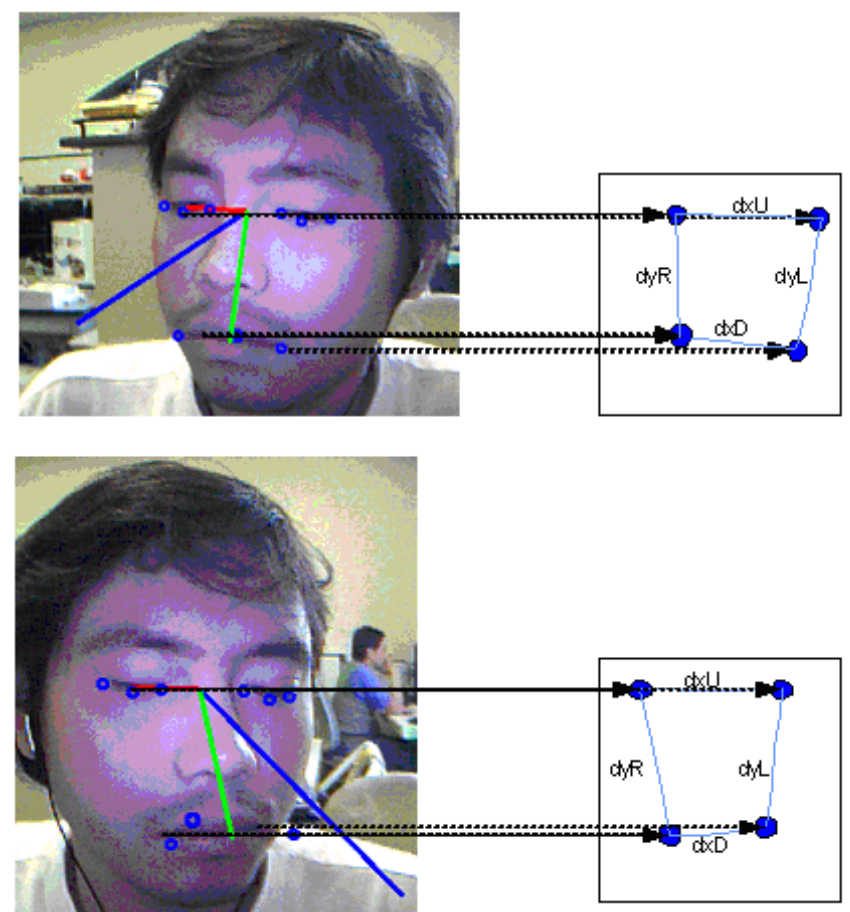

Fig. 13. Method for head pose angle estimation using the extracted feature points from the acquired face image

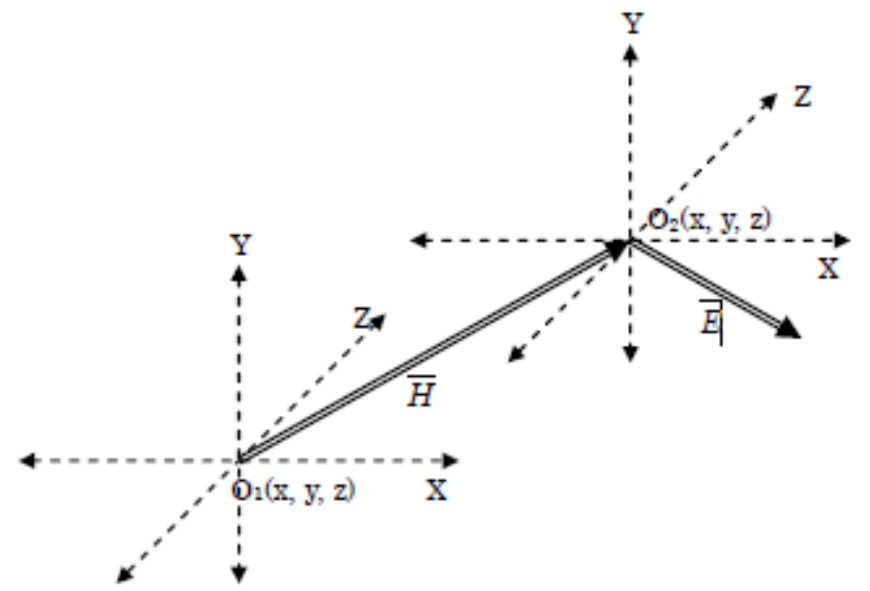

Fig. 14. Geometric relation between the rotation center of a head and an eyeball rotation center

Therefore, I need to get a user to maintain distance with a computer display to some extent (separating beyond the defined distance and twisting like). As also described before, to rotation of $\mathrm{x}$ of a head, the parallel translation of a $y$ direction, a roll, a pitch, and the direction of Yaw, it is detectable. As for parallel translation, it is highly accurate and can presume correctly about $100 \%$. Therefore, the degree of setting head rotation angle and average estimation error by five subjects were checked by experiment here. An experimental result is shown in Fig.15. Since the Yaw rotation of a head is rotation in a head plane, a presumed error is comparatively small. The presumed error of the direction of a pitch was the largest, and it turned out that the presumed error of about 5 times is produced also in rotation of about 1.0 degrees in the pitch rotation to a direction which especially bows. Even if it is relatively stout and rotates about 30 degrees to the pitch rotation to a direction which raises the head, the presumed error is settled in about 1.7 degrees. Moreover, the presumed error over rotation of the head of the roll direction was located in that middle, and when it was \pm 30 roll rotations, it has checked fitting in the presumed error of less than 1.7 degrees. Furthermore, one of eyes occlusion roll rotation exceeding 40 degrees, and a presumed error becomes large rapidly. Therefore, $\pm 30-10 \sim+30$ degrees, even if the head rotated \pm 30 degrees in a roll, a pitch, and each direction of Yaw, it checked fitting in the presumed error of less than 1.7 degrees to them.

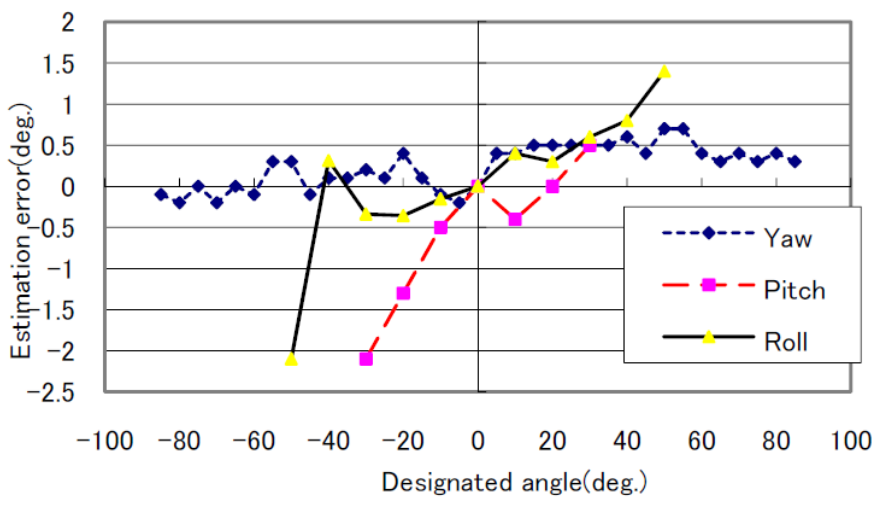

Fig. 15. Head angle estimation error

A cornea curvature radius is presumed using the Purkinje image of two points obtained by using the two-point light source proposed in this paper, the proposed method of eyeball rotation angle estimation which does not need the calibration for individual difference dissolution of cornea curvature are 0.57 degree and $\mathrm{y}$ to the direction of $\mathrm{x}$ in the state where the head was fixed in simple. It turned out that the degree of eyeball rotation angle can be presumed with the error of about 0.98 degree in a direction. Moreover, since the degree estimation method of head rotation angle using the details of the face proposed in this paper is the presumed error of about 1.7 degrees under restriction of the degree of rotation angle, it assumes that rotation of these heads and rotation of an eyeball are independent, and it is RSS: Root Sum Square is taken, it turns out that the eyeball angle estimation accuracy in the case of permitting rotation of a head is 1.988 degrees in a $\mathrm{x}$ direction at 1.821 degrees and a direction.

If a look is presumed and rotation is permitted for a head in the above-mentioned restriction range from the face picture acquired from the camera which separated $150 \mathrm{~mm}$, the direction of a look can be presumed in the error of about 2 times. When an intersection with a look is put on the computer 
display which left this $300 \mathrm{~mm}$, it is equivalent to about $10.472 \mathrm{~mm}$ look and a display intersection position estimation error. When a with a pixel size (4 pixels / 10mm) computer display is assumed, this intersection position estimation error will be equivalent to about 4 pixels. Fig.16 sets up a target on 7 pixels from a center as an example of look stability, gets five subjects to gaze at the target concerned for 30 seconds, and shows what plotted the presumed intersection position. Therefore, by making distance between adjacent keys into 10 pixels or more showed that rotation of a head was permitted and the degree estimation method of eyeball rotation angle which does not need a calibration could be realized.

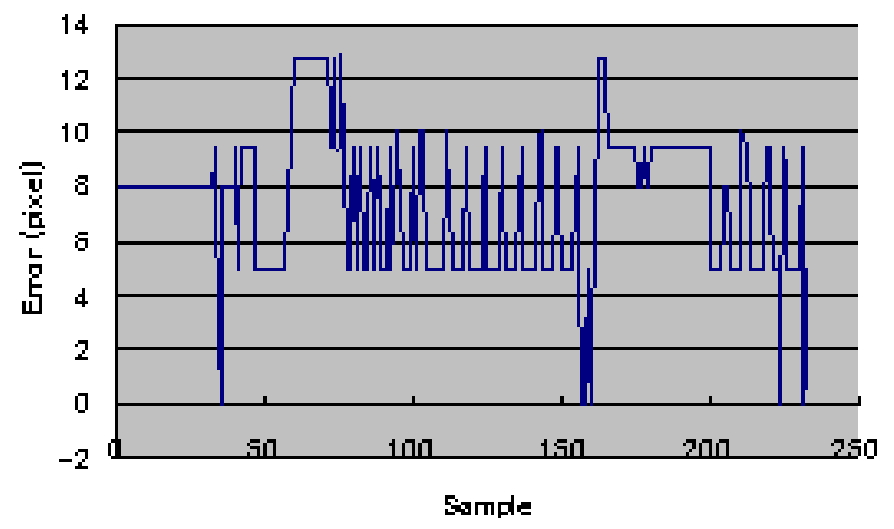

Fig. 16. Stability of the estimated location of fixed point

\section{Error Analysis}

In the experiment, eyeball angle estimation accuracy was checked by seeing five indices displayed on the display one by one, where a head is fixed simply. The presumed result of the eyeball angle detection from which a cornea curvature radius differs is shown in Fig.17. It is delta $\mathrm{x}$ and $\mathrm{y}$ when a cornea curvature radius is set to $\mathrm{R}=7.92 \mathrm{~mm}$ at this time. $\Delta \mathrm{xy}=\mathrm{it}$ is 1.128. Near the measured cornea curvature radius has the highest degree detection accuracy of eyeball rotation angle. Therefore, it has checked that rather than led which uses the measured cornea curvature radius to improvement in the degree detection accuracy of eyeball rotation angle using the standard value of a cornea curvature radius.

Change of the look estimation result of survey by head parallel translation and rotation is shown in Fig.18. There are two sets of the error analysis results, delta $x, y, z$ and mean of delta x,y,z. Fig.18 (a) and (b) show the two cases for cornea curvature radius estimation. On the other hand, the error analysis results for head angle estimation in directions of $\mathrm{x}, \mathrm{y}, \mathrm{z}$ are shown in Fig.18 (c), (d) and (e), respectively. Meanwhile, sensitivity analysis results for delta $\mathrm{x}, \mathrm{y}, \mathrm{z}$ and mean of delta $\mathrm{x}, \mathrm{y}, \mathrm{z}$ in directions of roll, pitch and yaw angles are shown in Fig.18 (f) to (i)

In measurement of a cornea curvature radius, it seems that the big accuracy fall has occurred when this is exceeded since it has measured in $3.0-5.0 \mathrm{~mm}$ from the cornea center-ofcurvature part. Moreover, in the influence on the presumed accuracy by the head rotation to the direction of a pitch, since the influence of eyelashes becomes large in downward rotation of a head, pupil detection becomes difficult (measurement is impossible), and since the influence of upward rotation of a head of eyelashes decreases, its pupil detection accuracy improves. From this, the camera position at the time of measurement is understood that it is desirable to install by arrangement which looks up at a head.

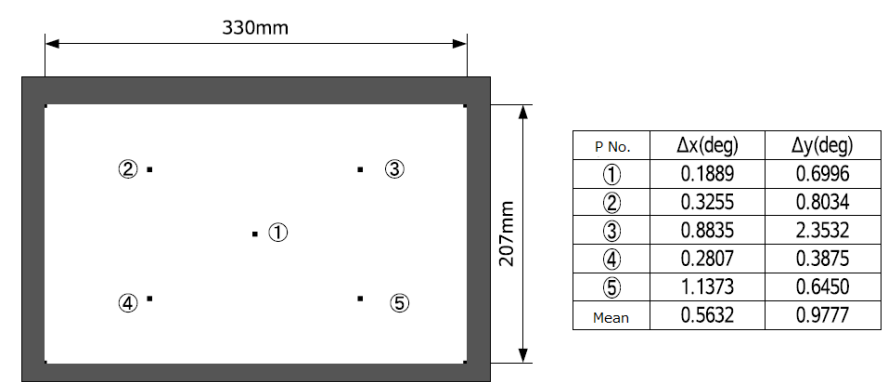

Fig. 17. presumed result of the eyeball angle detection from which a cornea curvature radius differs

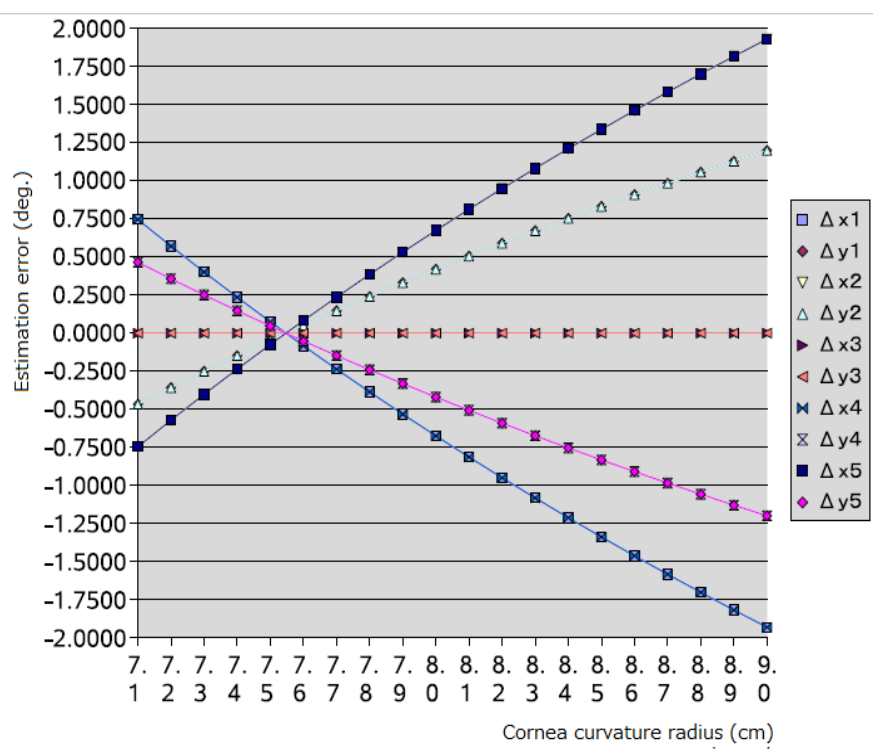

(a)Cornea curvature radius

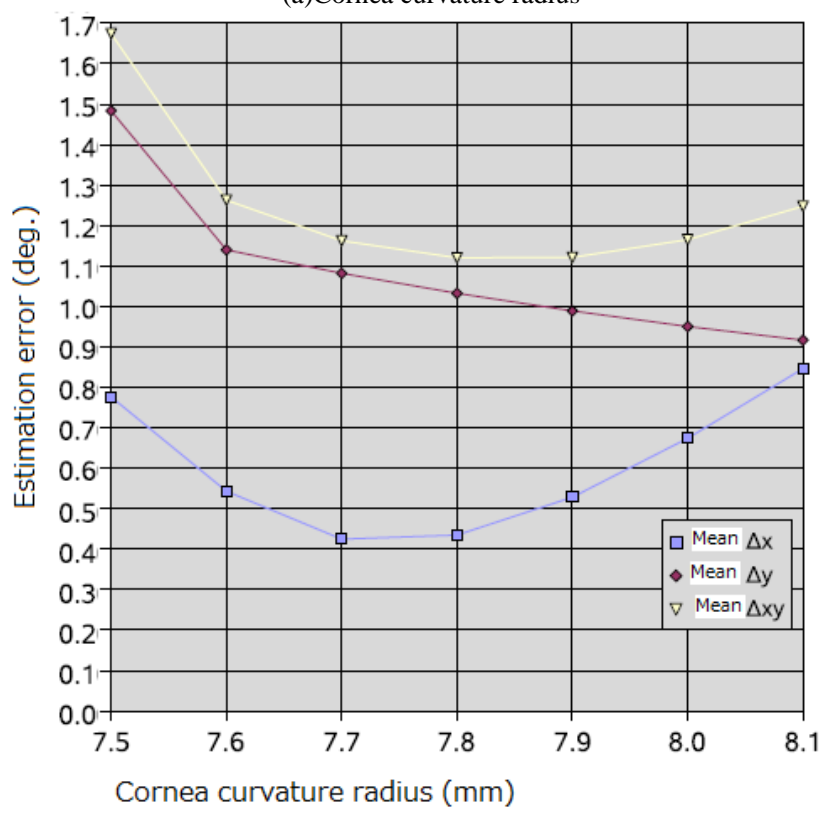

(b)Cornea curvature radius 


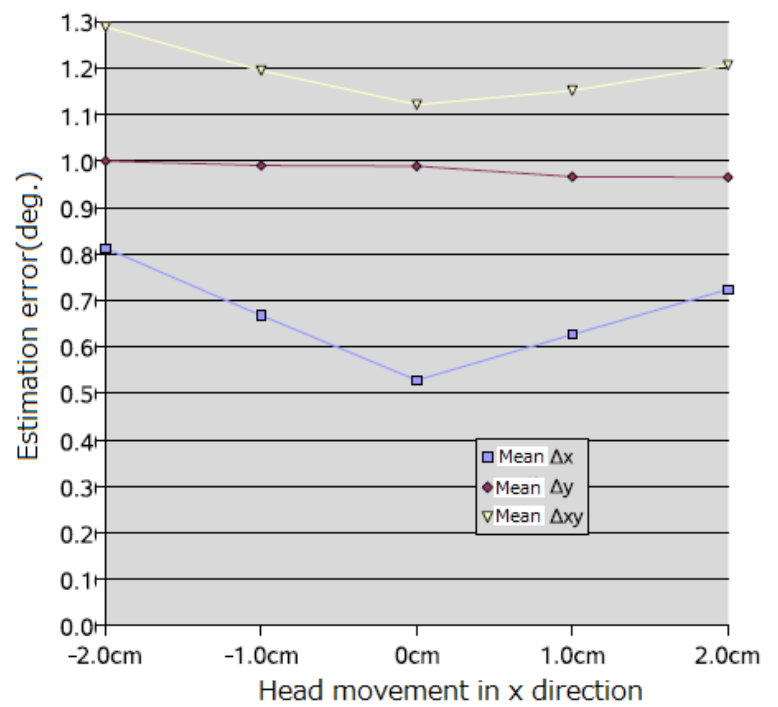

(c) $\mathrm{x}$

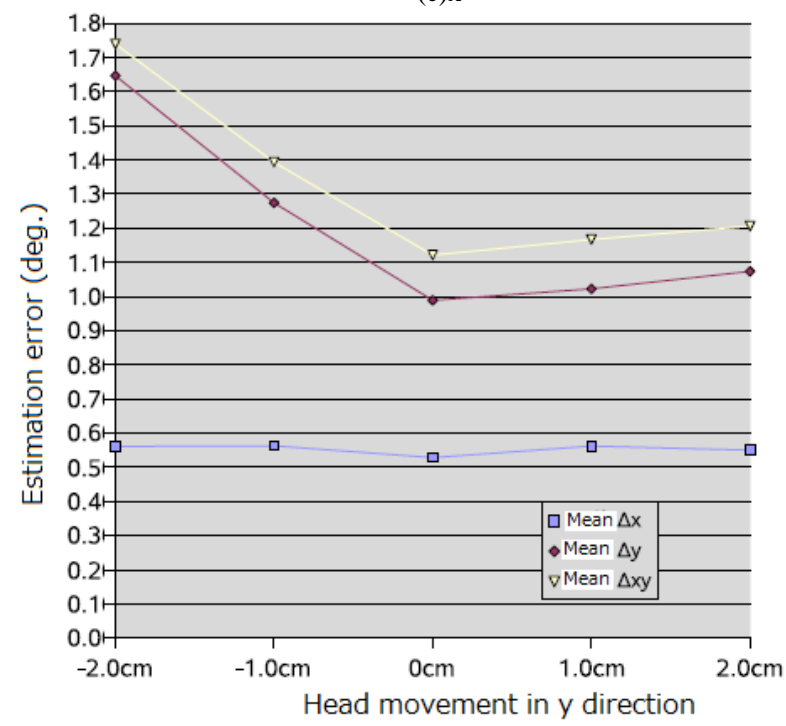

(d)y

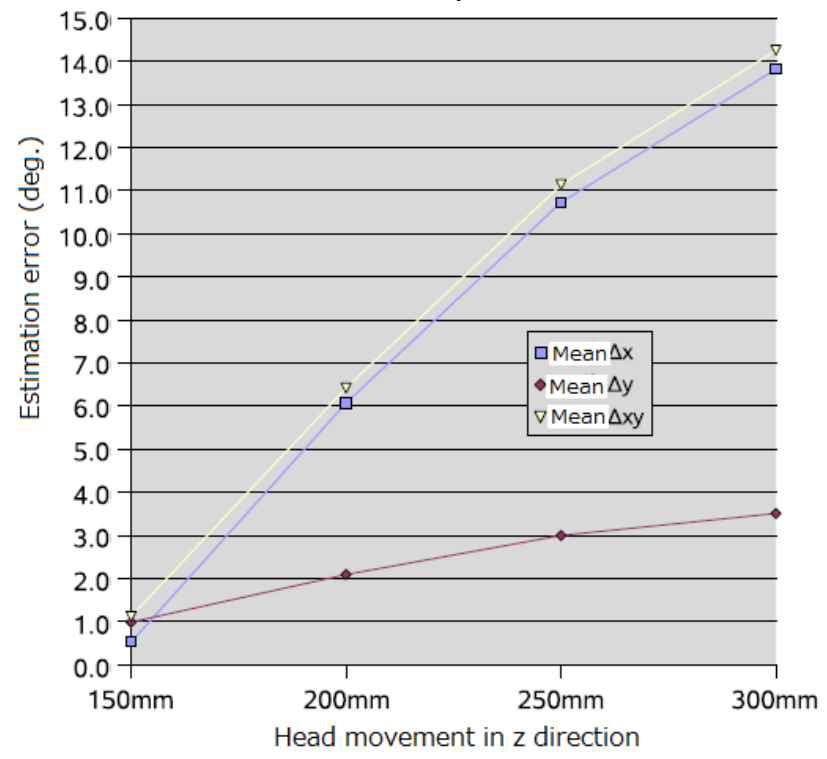

(e) $z$

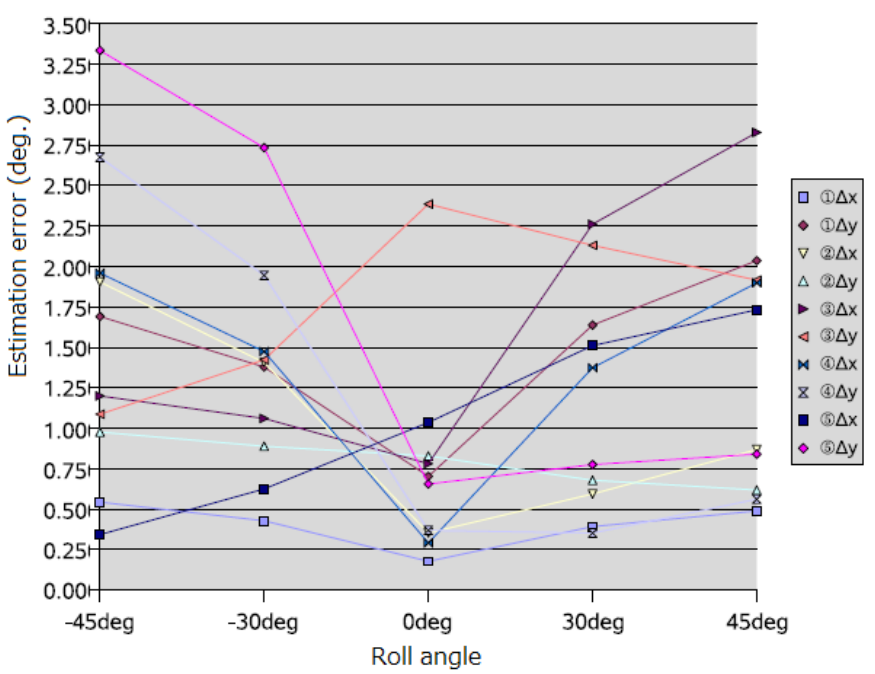

(f)Roll

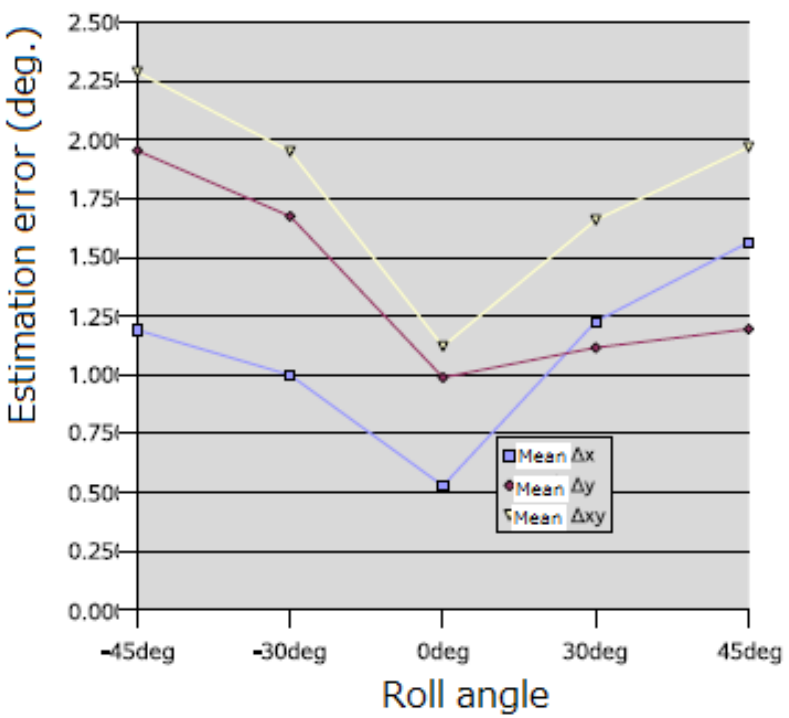

(g)Roll

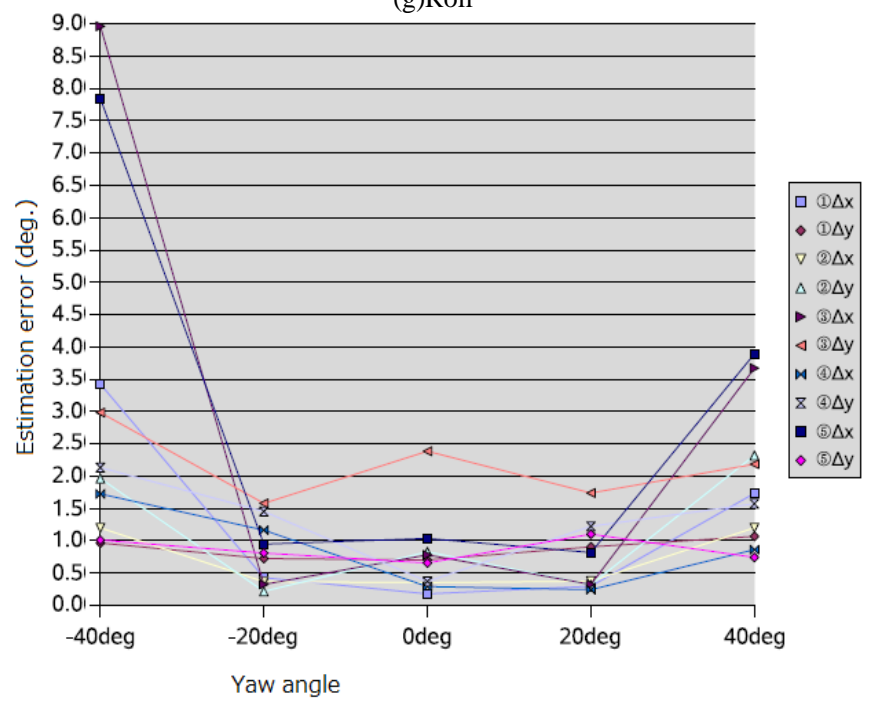

(h)Yaw 


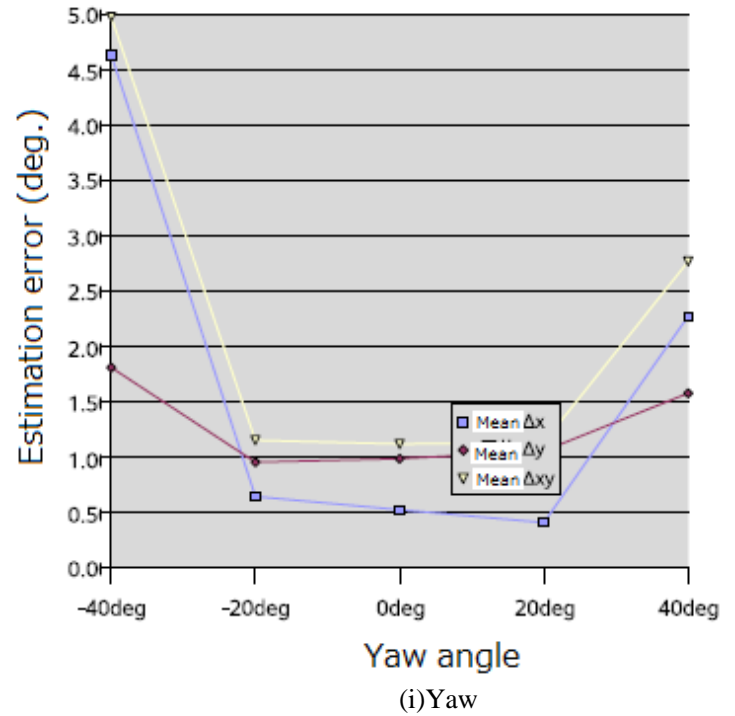

Fig. 18. Results from the Error Analysis

\section{CONCLUSION}

Error analysis of line of sight estimation using Purkinje images for eye-based Human-Computer Interaction: HCI is conducted. Double Purkinje images which are obtained by two points of light sources are used in the proposed method for eye rotation angle estimation. It aimed at the improvement of the eyeball rotation angle accuracy by simply presuming the value of the curvature radius of the cornea. This technique is a cancellation of the time of the calibration that is the problem of past glance presumption. By presuming the size of the radius of curvature of the cornea. As a result, the eyeball rotation angle presumption accuracy of about $0.98 \mathrm{deg}$ was obtained $0.57 \mathrm{deg}$ and horizontally in the vertical direction without doing the calibration.

This paper proposed the estimation method of eyeball rotation angle which does not need the calibration for individual difference dissolution of cornea curvature by presuming a cornea curvature radius using the Purkinje image of two points obtained by using a two-point light source. As a result, in the state where the head was fixed in simple, they are $0.98 \mathrm{deg}(\mathrm{s})$ to $0.57 \mathrm{deg}(\mathrm{s})$ and the direction of $\mathrm{y}$ in the direction of $\mathrm{x}$. The degree estimation accuracy of eyeball rotation angle was acquired with the error of a grade. Moreover, by the angle estimation method of the plane defined by the details of the face proposed since rotation of a head was permitted, when preparing restriction in each rotation of a roll, a pitch, and a yaw direction, it checked that the degree of rotation angle could be presumed with the error of about 0.5 degree. When it assumed that rotation of these heads and rotation of an eyeball were independent, it turned out that the eyeball angle estimation accuracy in the case of permitting rotation of a head is 1.988 degrees in a $\mathrm{x}$ direction at 1.821 degrees and a $\mathrm{y}$ direction. That is, since the degree of viewing angle beyond this error was detectable, when detaching and setting up the interval of a key rather than this, it turned out that it can guarantee that discernment of a key is possible.

\section{ACKNOWLEDGEMENTS}

Author would like to thank Mr. Makoto Yamaura of former student of Saga University for his efforts for conducting the experiments.

\section{REFERENCES}

[1] K. Matsuda, T. Nagami and S. Yamane, Development of gaze measurement system, Technical Report of the Institute of Electronics, Information and Communication Engineers, TL2002-2, 2000.

[2] T. Ohno, N. Takegawa, and A. Yoshikawa, Gaze estimation method based on eyeball shape model, Proc. of the 8th Image Sensing Symposium, pp.307-312, 2002.

[3] Y. Ebisawa, 3D gaze measurement equipment, Japanese Patent No.2005-198743, 2005.

[4] H. Tanaka, S. Hikita, K. Kasai, and A. Takeda, Gaze estimation method based on cornea curvature center estimation with two light sources, Journal of the Institute of Electronics, Information and Communication Engineers, 108, 479, MBE2008-128, 117-180, 2009.

[5] Kohei Arai and Kenro Yajima, Communication Aid and Computer Input System with Human Eyes Only, Electronics and Communications in Japan, Volume 93, Number 12, 2010, pages 1-9, John Wiley and Sons, Inc., 2010.

[6] Kohei Arai, Computer Input by Human Eyes Only and Its Applications, Intelligent Systems in Science and Information, 2014, Studies in Computer Intelligence, 591, 1-22, Springer Publishing Co. Ltd., 2015.

[7] Kohei Arai, Makoto Yamaura, Blink detection accuracy improvement by means of morphologic filter in designated key selection and determination for computer input by human eyes only. Journal of Image Electronics Society of Japan, 37, 5, 601-609, 2008

[8] Kohei Arai, Kenro Yajima, Communication aid system using computer input by human eyes only, Journal of Electric Society of Japan, Transaction C, 128-C,11,1679-1686, 2008

[9] Kohei Arai, Kenro Yajima, Communication aid system using computer input by human eyes only, Journal of Electric Society of Japan, Transaction C, 128-C, 11,1679-1686, 2008

[10] Djoko Purwanto, Ronny Mardiyanto, Kohei Arai, Electric wheel chair control with gaze detection and eye blinking, Proceedings of the International Symposium on Artificial Life and Robotics, GS9-4, 2009

[11] Djoko Purwanto, Ronny Mardiyanto and Kohei Arai, Electric wheel chair control with gaze detection and eye blinking, Artificial Life and Robotics, AROB Journal, 14, 694,397-400, 2009.

[12] Kohei Arai, Ronny Mardiyanto, Computer input by human eyes only with blink detection using Gabor filter, Journal of Visualization Society of Japan, 29, Suppl.2, 87-90, 2009

[13] Kohei Arai and Makoto Yamaura, Computer input with human eyes only using two Purkinje images which works in a real time basis without calibration, International Journal of Human Computer Interaction, 1, 3, 71-82, 2010

[14] Kohei Arai, Ronny Mardiyanto, A prototype of electric wheel chair control by eye only for paralyzed user, Journal of Robotics and Mechatronics, 23, 1, 66-75, 2010.

[15] Kohei Arai, Kenro Yajima, Robot arm utilized having meal support system based on computer input by human eyes only, International Journal of Human-Computer Interaction, 2, 1, 120-128, 2011

[16] Kohei Arai, Ronny Mardiyanto, Autonomous control of eye based electric wheel chair with obstacle avoidance and shortest path finding based on Dijkstra algorithm, International Journal of Advanced Computer Science and Applications, 2, 12, 19-25, 2011.

[17] Kohei Arai, Ronny Mardiyanto, Eye-based human-computer interaction allowing phoning, reading e-book/e-comic/e-learning, Internet browsing and TV information extraction, International Journal of Advanced Computer Science and Applications, 2, 12, 26-32, 2011

[18] Kohei Arai, Ronny Mardiyanto, Eye based electric wheel chair control system-I(eye) can control EWC-, International Journal of Advanced Computer Science and Applications, 2, 12, 98-105, 2011. 
[19] Kohei Arai, Ronny Mardiyanto, Evaluation of users' impact for using the proposed eye based HCI with moving and fixed keyboard by using eeg signals, International Journal of Research and Reviews on Computer Science, 2, 6, 1228-1234, 2011.

[20] Kohei Arai, Ronny Mardiyanto, Electric wheel chair controlled by human eyes only with obstacle avoidance, International Journal of Research and Reviews on Computer Science, 2, 6, 1235-1242, 2011.

[21] K.Arai, R.Mardiyanto, Evaluation of users' impact for using the proposed eye based HCI with moving and fixed keyboard by using eeg signals, International Journal of Research and review on Computer Science, 2, 6, 1228-1234, 2012.

[22] K.Arai, R.Mardiyanto, Electric wheel chair controlled by human eyes only with obstacle avoidance, International Journal of Research and Review on Computer Science, 2, 6, 1235-1242, 2012.

[23] Kohei Arai, R.Mardiyanto, Robot arm control with human eyes only and its application to help having meal for patients, Journal of Electrical Engineering Society of Japan, Transaction C, C132, 3, 416-423, 2012.

[24] Kohei Arai, Human-Computer Interaction with human eyes only and its applications, Journal of Image Electronics Society of Japan, 41, 3, 296301, 2012.

[25] R.Mardiyanto, K.Arai, Eye-based Human Computer Interaction (HCI) A new keyboard for improving accuracy and minimizing fatigue effect, Scientific Journal Kursor, (ISSN 0216-0544), 6, 3, 1-4, 2012.

[26] K.Arai, R.Mardiyanto, Moving keyboard for eye-based Human Computer Interaction: HCI, Journal of Image and Electronics Society of Japan, 41, 4, 398-405, 2012.

[27] Kohei Arai, R.Mardiyanto, Service robot which is controlled by human eyes only with voice communication capability, Journal of Image Electronics Society of Japan, 41, 5, 535-542, 2012.

[28] Kohei Arai, Ronny Mardiyanto, Eye-based domestic robot allowing patient to be self-services and communications remotely, International Journal of Advanced Research in Artificial Intelligence, 2, 2, 29-33, 2013.

[29] Kohei Arai, Ronny Mardiaynto, Method for psychological status estimation by gaze location monitoring using eye-based HumanComputer Interaction, International Journal of Advanced Computer Science and Applications, 4, 3, 199-206, 2013.

[30] Kohei Arai, Kiyoshi Hasegawa, Method for psychological status monitoring with line of sight vector changes (Human eyes movements) detected with wearing glass, International Journal of Advanced Research in Artificial Intelligence, 2, 6, 65-70, 2013
[31] Kohei Arai, Wearable computing system with input output devices based on eye-based Human Computer Interaction: HCI allowing location based web services, International Journal of Advanced Research in Artificial Intelligence, 2, 8, 34-39, 2013.

[32] Kohei Arai Ronny Mardiyanto, Speed and vibration performance as well as obstacle avoidance performance of electric wheel chair controlled by human eyes only, International Journal of Advanced Research in Artificial Intelligence, 3, 1, 8-15, 2014.

[33] Kohei Arai, Service robot with communicational aid together with routing controlled by human eyes, Journal of Image Laboratory, 25, 6, 24-29, 2014

[34] Kohei Arai, Information collection service system by human eyes for disable persons, Journal of Image Laboratory, 25, 11, 1-7, 2014

[35] Kohei Arai, Relations between psychological status and eye movements, International Journal of Advanced Research on Artificial Intelligence, 4, 6, 16-22, 2015.

\section{AUTHORS PROFILE}

Kohei Arai, He received BS, MS and PhD degrees in 1972, 1974 and 1982, respectively. He was with The Institute for Industrial Science and Technology of the University of Tokyo from April 1974 to December 1978 and also was with National Space Development Agency of Japan from January, 1979 to March, 1990. During from 1985 to 1987, he was with Canada Centre for Remote Sensing as a Post Doctoral Fellow of National Science and Engineering Research Council of Canada. He moved to Saga University as a Professor in Department of Information Science on April 1990. $\mathrm{He}$ was a councilor for the Aeronautics and Space related to the Technology Committee of the Ministry of Science and Technology during from 1998 to 2000. He was a councilor of Saga University for 2002 and 2003. He also was an executive councilor for the Remote Sensing Society of Japan for 2003 to 2005. He is an Adjunct Professor of University of Arizona, USA since 1998. $\mathrm{He}$ also is Vice Chairman of the Commission-A of ICSU/COSPAR since 2008. He received Science and Engineering Award of the year 2014 from the minister of the ministry of Science Education of Japan and also received the Bset Paper Award of the year 2012 of IJACSA from Science and Information Organization: SAI. In 2016, he also received Vikram Sarabhai Medal of ICSU/COSPAR and also received 20 awards. He wrote 34 books and published 520 journal papers. He is Editor-in-Chief of International Journal of Advanced Computer Science and Applications as well as International Journal of Intelligent Systsems and Applications. http://teagis.ip.is.sagau.ac.jp/ 